\title{
Analysis of faster optical response in core/shell nanocrystals ferroelectric liquid crystal composite
}

\author{
S. Pandey, ${ }^{1}$ D.P. Singh, ${ }^{1,2}$ Atul Srivastava, ${ }^{1}$ and R. Manohar ${ }^{* 1}$ \\ ${ }^{1}$ Liquid Crystal Research Lab, Physics Department, University of Lucknow, Lucknow, India-226007 \\ ${ }^{2}$ Unité de Dynamique et Structure des Matériaux Moléculaires, EA 4476, Université du Littoral Côte d'Opale, \\ F-59140 Dunkerque, France.
}

Received November 09, 2015; accepted December 27, 2015; published December 31, 2015

\begin{abstract}
In the present study $\mathrm{Cd}_{1-\mathrm{x}} \mathrm{Zn}_{\mathrm{x}} \mathrm{S} / \mathrm{ZnS}$ core/shell nanocrystals dispersed ferroelectric liquid crystal (FLC) has been examined. The effect of NCs concentrations in FLC matrix for the electro-optical applications has been studied in the $\mathrm{SmC}^{*}$ phase. A considerable variation in the parameters like tilt angle, spontaneous polarization, response time, anchoring energy and rotational viscosity has been observed for the composite system. Almost twofold faster optical response for the composite system is one of the promising results of the present study. Thus the present composite can be used for faster electro-optical devices.
\end{abstract}

In the last few years, nanocrystals (NCs) have attracted a great deal of attention of the scientific community as an important dopant for the LC matrix. The number of improvements brought by the dispersion of NCs into liquid crystal (LC) can be used in electro-optical (EO) devices. The application of nanocrystals to examine various important phenomena has proved them to be the beneficiary entrant owing to their tunable electrical, optical and electro-optical parameters [1-3]. The interaction between ferroelectric liquid crystal (FLC) and NCs suggests that NCs can be dispersed well in a small concentration with reduced switching time and operating voltage [4]. However, a higher concentration of NCs causes aggregation and therefore produces an adverse effect on the properties of LC as reported by Singh et al. [5]. Recent studies on different FLC-NC composites, reveal that these composites may demonstrate significant changes in the electro-optical parameters. The present paper extends the knowledge of studying the effect of concentration of NCs on various electro-optical properties of the FLC-NC composite.

The FLC used in the present work is Felix 17/000 purchased from Clariant Chem. Co. Ltd., Germany. The phase transition scheme of FLCs is as follows:

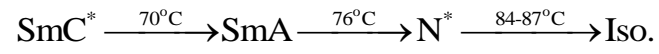

\footnotetext{
*E-mail: rajiv.manohar@gmail.com
}

$\mathrm{Cd}_{1-\mathrm{x}} \mathrm{Zn}_{\mathrm{x}} \mathrm{S} / \mathrm{ZnS}$ core/shell NCs having diameter $8.7 \mathrm{~nm}$ [6] have been dispersed in 0.5 and $1.0 \mathrm{wt} / \mathrm{wt} \%$ concentrations in the pure FLC. To insure the homogeneous dispersion of NCs, the mixtures were homogenized for 1 hour in the ultrasonic mixer. In order to prepare the sample holders, Indium Tin Oxide (ITO) coated glass plates have been used in the present study. A nylon 6/6 polymer layer was applied on the ITO coated glass plates at which unidirectional rubbing was done in order to achieve the planar alignment. The thickness of the sample holders $(5 \mu \mathrm{m})$ was achieved by using the Mylar spacer. The electro-optical measurements have been performed at $35^{\circ} \mathrm{C}$ by applying triangular and square wave pulses using a programmable function generator (Tektronix, AFG-3021B). The electrical signals $\left(20 \mathrm{~V}_{\mathrm{PP}}\right.$, $10 \mathrm{~Hz}$ ) were applied to the photo detector (Instec PD02L1). The tilt angle was obtained by setting two extinction positions of the sample. The tilt angle is half of the angle between the two extinction positions [4]. To measure the spontaneous polarization (Ps) of the pure and the composite system, a polarization reversal current method has been used [4]. The optical response $(\tau)$ of the samples was recorded on a digital storage oscilloscope (Tektronix, TDS-2024C). The detailed experimental information for electro-optical measurements has been reported by our group [7-9]. In $\mathrm{SmC}^{*}$ phase, the rotational viscosity $(\gamma)$ for a cone motion was calculated using the relation:

$$
\gamma=\frac{P_{S} \tau E}{1.8}
$$

Figure 1 shows the variation of tilt angle $(\theta)$ with concentration of a dopant in the $\mathrm{SmC}^{*}$ phase at $20 \mathrm{~V}$ and $35^{\circ} \mathrm{C}$. The change in the molecular ordering and alignment depends upon the concentration of NCs in pure FLC. The Fig. 1 depicts a decrease in the value of the tilt angle with increasing concentration of NCs. The dispersion of NCs into the pure FLC suppresses the conical geometry of the composite attributable to the interaction between the FLC and NCs and between that of NCs [4]. Thus, the suppressed conical geometry of the composite decreases 
the tilt angle for the composite in comparison to that of pure FLC.

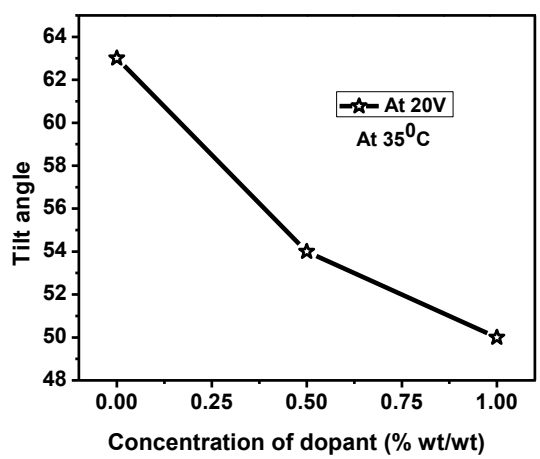

Fig. 1. Variation of tilt angle with concentration of NCs.

The variation of spontaneous polarization (Ps) with the concentration of NCs at $35^{\circ} \mathrm{C}$ is shown in Fig. 2. It is clear from Fig. 2 that Ps has decreased on addition of NCs. It is known from the literature that Ps is the secondary order parameter whereas tilt angle is the primary order parameter for $\mathrm{SmC}^{*}$ phase [10]. The decrease in the value of Ps for the composite system can be accredited to the decrease in the value of the tilt angle. Another cause behind the decrease in Ps may be attributed to the decrease in the effective dipole moment of the composite system due to the presence of NCs. The inset shows electrical response for the measurement of Ps.

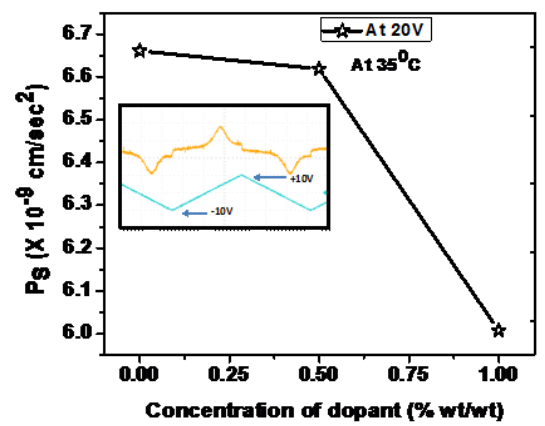

Fig. 2. Variation of Ps with concentration of NCs.

Figure 3 represents the variation of rotational viscosity $(\gamma)$ with concentration of $\mathrm{NCs}$ at $35^{\circ} \mathrm{C}$. It is known that insertion of any type of dopant increases the rotational viscosity of the composite system. However, in the present study, it is found that the rotational viscosity has decreased with increasing concentration of NCs. The cause of decrease in rotational viscosity for the composite is linked with the decrease in molecular ordering which occurs on addition of NCs into the pure FLC. NCs distribute in pure FLC matrix in a random manner which disturbs the FLC geometry which effects molecular ordering in FLC. Thus the rotational viscosity of the composite system decreases.

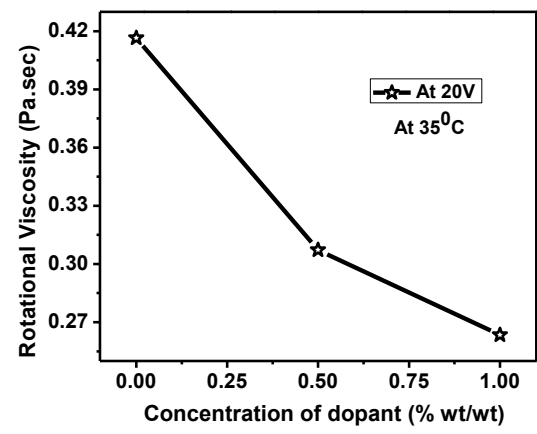

Fig. 3. Variation of rotational viscosity with concentration of NCs.

The variation of dispersion $\left(\mathrm{W}_{\mathrm{D}}\right)$ and polarization anchoring $\left(\mathrm{W}_{\mathrm{P}}\right)$ energy coefficients with concentration of a dopant has been shown in Fig. 4. It is known that $\mathrm{W}_{\mathrm{D}}$ proportional to $\tau^{-1}$ whereas $\mathrm{W}_{\mathrm{P}}$ is proportional to $\mathrm{P}_{\mathrm{S}}^{1 / 2}$ respectively [11]. A remarkable reduction in both the anchoring energy coefficients has been observed. The reduction in anchoring energy coefficients is analogous to a decrease in the order parameter of the composite.
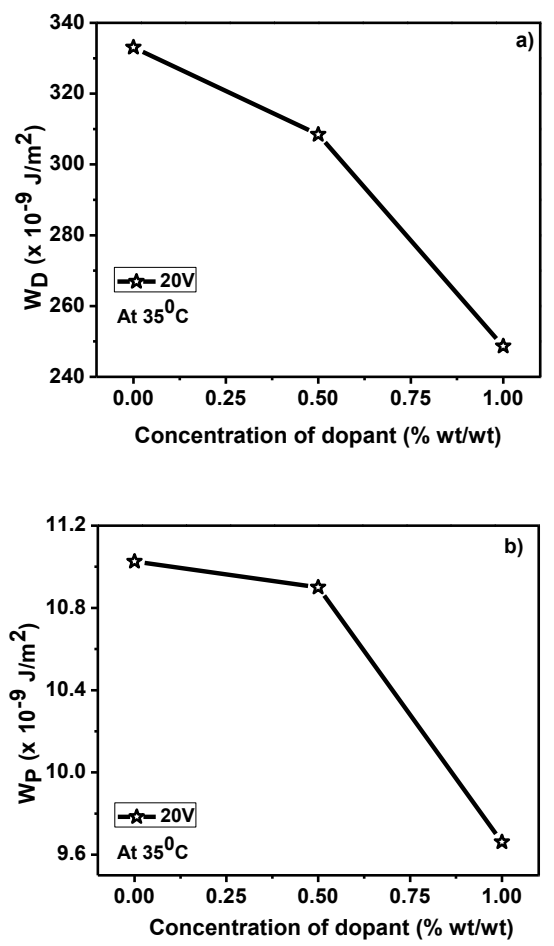

Fig. 4. Variation of a) $W_{D}$ and b) $W_{P}$ with concentration of NCs.

(C) 2015 Photonics Society of Poland 
The response time is a crucial parameter for display applications. The variation of response time with concentration of dopant at $35^{\circ} \mathrm{C}$ is given in Fig. 5. It is shown in the Fig. 5 that the response time becomes faster on addition of NCs as compared to that of pure FLC. The higher optical response time of the composite is credited to the change in dispersion anchoring energy of pure FLC due to the addition of QDs. The inset shows the optical response corresponding to the input square wave of amplitude $20 \mathrm{Vpp}$.

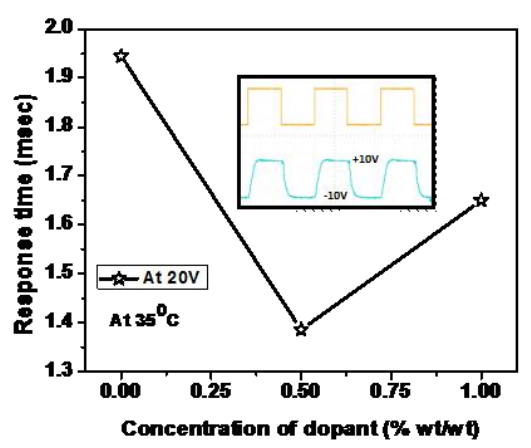

Fig. 5. Variation of response time with concentration of NCs.

In short, the effect of concentration of $\mathrm{Cd}_{1-\mathrm{x}} \mathrm{Zn}_{\mathrm{x}} \mathrm{S} / \mathrm{ZnS}$ NCs on the electro-optical parameters of Felix 17/000 has been investigated in the present work. The addition of NCs into pure FLC provides an efficient way of altering the electro-optical properties of the FLC. The outcome of the present investigation provides a better understanding of the influence of the semiconductor QDs doping on various properties of FLC. The decrease in various EO parameters like $\theta$, Ps, $\gamma, \tau$ and anchoring energy of the composite system has been explained on the basis of molecular interactions among NCs and FLC. The composite system with faster response makes them compatible for electro optical displays. Consequently, the dispersion of NCs into the pure FLC provides new LC mixtures with better quality for the upcoming generation of display technology.

Authors are thankful to DST for INDO-POLISH project grant. One of the authors SP is sincerely thankful to UGC, New Delhi for providing financial assistance in the form of UGC-BSR Fellowship. DPS is also thankful to CEFIPRA for Raman-Charpak fellowship in France. RM is thankful to UGC for grant of research award (2014-16).

\section{References}

[1] J. Mirzaei, M. Urbanski, K. Yu, H. S. Kitzerow, T. Hegmann, J. Mater Chem. 21, 12710 (2011).

[2] S. Ghosh et al., EPL 96, 47003 (2011).

[3] A. Chaudhary, P. Malik, R. Mehra, K.K. Raina, Phase Trans. 85, 244 (2012).

[4] S. Pandey et al., Liq. Cryst. 41, 1811 (2014).

[5] D.P. Singh, S.K. Gupta, R. Manohar, Adv. Condens. Matt. Phys. 2013, 1 (2013)

[6] W.K. Bae, M.K. Nam, K. Char, S. Lee, Chem. Mater. 20, 5307 (2008).

[7] S. Pandey et al., J. Mol. Liq. 211, 157 (2015).

[8] D.P. Singh, S.K. Gupta, R. Manohar, Liq. Cryst. 42, 1159 (2015).

[9] A.K. Srivastava, A.K. Misra, P.B. Chand, R. Manohar, J.P. Shukla, Phys. Lett. A 371, 490 (2007).

[10] Yu.P. Panarin, Yu.P. Kalmykov, S.T.M. Laughadha, H. Xu, J. K. Vij, Phys. Rev. E 50, 4763 (1994).

[11] R. Manohar, A.K. Srivastava, P.K. Tripathi, D.P. Singh, J. Mater Sci. 46, 5969 (2011). 